\title{
Time Flies When We Intend to Act: Temporal Distortion in a Go/No-Go Task
}

\author{
Yoshiko Yabe ${ }^{1,2}$ and Melvyn A. Goodale ${ }^{1}$ \\ ${ }^{1}$ Brain and Mind Institute and the Department of Psychology, University of Western Ontario, London, Ontario, Canada, N6A 5B7, and ${ }^{2}$ Research Institute, \\ Kochi University of Technology, Kami, Kochi, 782 8502, Japan
}

\begin{abstract}
Although many of our actions are triggered by sensory events, almost nothing is known about our perception of the timing of those sensory events. Here we show that, when people react to a sudden visual stimulus that triggers an action, that stimulus is perceived to occur later than an identical stimulus that does not trigger an action. In our experiments, participants fixated the center of a clock face with a rotating second hand. When the clock changed color, they were required to make a motor response and then to report the position of the second hand at the moment the clock changed color. In Experiment 1, in which participants made a target-directed saccade, the color change was perceived to occur $59 \mathrm{~ms}$ later than when they maintained fixation. In Experiment 2, in which we used a go/no-go paradigm, this temporal distortion was observed even when participants were required to cancel a prepared saccade. Finally, in Experiment 3 , the same distortion in perceived time was observed for both go and no-go trials in a manual task in which no eye movements were required. These results suggest that, when a visual stimulus triggers an action, it is perceived to occur significantly later than an identical stimulus unrelated to action. Moreover, this temporal distortion appears to be related not to the execution of the action (or its effect) but rather to the programming of the action. In short, there seems to be a temporal binding between a triggering event and the triggered action.
\end{abstract}

Key words: eye movement; go/no-go; intentional binding; sense of agency; time perception

\section{Introduction}

A number of studies have shown that a sensory event can be shifted backward in time toward the action that was perceived to have caused that event (Haggard et al., 2002; Haggard and Clark, 2003; Wohlschläger et al., 2003; Buehner and Humphreys, 2009; Kawabe et al., 2013). It has been suggested that this temporal binding between an action and the event depends on a sense of agency, i.e., the subjective awareness that one is initiating, executing, and controlling one's actions and thus plays a causal role in the effects of those actions (Haggard and Chambon, 2012). However, all of this work has focused on self-paced actions and stimulus events that follow the performance of those actions, but in everyday life, many of our actions are not self-paced but are triggered instead by external events that of necessity precede the action. When we react to an external event, is our perception of the timing of that event the same as that of a passive observer? For example, in a baseball game, do the pitcher, the batter, and the audience all share the same temporal perception of how the events unfold? In this example, for all the players, except the

\footnotetext{
Received Oct. 21, 2014; revised Feb. 19, 2015; accepted Feb. 22, 2015.

Author contributions:Y.Y. and M.A.G. designed research;Y.Y.and M.A.G. performed research;Y.Y. analyzed data; Y.Y. and M.A.G. wrote the paper.

This research was supported by Natural Sciences and Engineering Research Council of Canada Grant 6313 (M.A.G.) and Japan Society for the Promotion of Science (KAKENHI) Grant 25750265 (Y.Y.).

The authors declare that they have no competing financial interests.

Correspondence should be addressed to Yoshiko Yabe, Brain and Mind Institute, Natural Science Center, University of Western Ontario, 1151 Richmond Street, London, Ontario, Canada, N6A 5B7. E-mail: yyabe@uwo.ca.

DOI:10.1523/JNEUROSCI.4386-14.2015

Copyright $\odot 2015$ the authors $\quad 0270-6474 / 15 / 355023-07 \$ 15.00 / 0$
}

pitcher, the actions are the consequence of external events rather than being the cause of such events. Remarkably, there have been no investigations focusing on the perceived timing of the stimulus events that trigger actions. However, such investigations are critical because, if there were temporal binding between stimulus events and the actions they precipitate, then the notion that temporal binding always depends on a sense of agency would have to be reexamined.

To investigate the perceived timing of stimulus events that trigger actions, we performed a series of experiments involving both eye movements and manual responses. We used a clock display with a rotating "second hand" first devised by Libet et al. (1983) to study the subjective onset of the intention to make an action. Participants were required to fixate the center of the clock and to report the position of the second hand at the exact moment the triggering stimulus occurred. For both eye movements and manual responses, we found that there was a systematic delay in the perceived onset of the visual cue that triggered the actions compared with situations in which no action was required and the onset of the stimulus was observed passively. Moreover, by using a go/no-go paradigm, we were able to show that this temporal distortion was also present on trials in which participants canceled the action. Together, these results suggest that there is a temporal binding between a stimulus event and the action it triggers and that the temporal delay that reflects this binding is related to the programming of the action and not its actual execution. Because the onset of the visual cue in our experiments preceded rather than followed the action, any account of tempo- 
ral distortion based on sense of agency would have to be modified or extended.

\section{Materials and Methods}

\section{Participants}

All participants had normal or corrected-to-normal vision. The protocol was approved by the Ethics Review Board of the University of Western Ontario. All participants gave informed written consent before taking part in the experiments.

\section{Apparatus}

All experiments were performed in a dark room. A chin rest was used to stabilize the head. Stimuli were presented on a CRT monitor (G90f; ViewSonic) with a refresh rate of $100 \mathrm{~Hz}$ at a resolution of $1024 \times 768$ pixels and were viewed binocularly at the viewing distance of $53 \mathrm{~cm}$. The presentation of the stimulus was controlled by software written in MATLAB 8.1.0.604 (MathWorks) assisted by Psychtoolbox 3.0.9 (Brainard, 1997; Pelli, 1997).

\section{Eye-movement recording and analysis}

Eye movements were recorded using an EyeLink 1000 Tower Mount system (SR Research) with a sampling rate of $1000 \mathrm{~Hz}$. Eye-movement recordings were analyzed offline using custom software. The system detected the start and end of a saccade when eye velocity exceeded or fell below $35 \%$ s. Trials were excluded from the analysis if the participant blinked or made a saccade when the clock changed color $(0.48 \%$ of all trials in Experiment 1, 0.83\% of all trials in Experiment 2).

We excluded trials if the participant made a saccade before $640 \mathrm{~ms} \mathrm{(a}$ quarter rotation of the clock) had elapsed after the color change in the control condition of Experiment 1 (13.45\% of all trials) or in the no-go trials (31.11\% of all trials) and the control condition ( $18.40 \%$ of all trials) of Experiment 2. The high rate of "error" saccades on no-go and control trials is to be expected, particularly in the no-go trials in which participants have to cancel their movement plan. We also excluded trials if participants did not make a saccade in the saccade condition of Experiment $1(0.95 \%$ of all trials $)$ or in the go trials of Experiment $2(0.29 \%$ of all trials). Saccades with a very short delay $(<100 \mathrm{~ms})$ were excluded ( $0.68 \%$ of all trials in Experiment 1, none of the trials in Experiment 2). Saccades with a very long delay $(>640 \mathrm{~ms})$ were also excluded $(5.58 \%$ of all trials in Experiment 1, 7.52\% of all trials in Experiment 2). Overall, $\sim 70 \%$ of the trials were used in the final analyses of Experiments 1 and 2. Trials in which participants broke fixation after the change in color of the clock in Experiment 3A (28.14\% of all trials) were excluded from the analysis. Eye movements were not recorded in Experiment 3B.

\section{Hand-movement recording}

In Experiment 3A, participants released a depressed Enter key on a "silent" keyboard (REALFORCE91UBK-S; Topre). Trials were excluded from the analysis if the participant released the key in the control condition or no-go trials (7.22 and $31.30 \%$, respectively). Trials were also excluded from the analysis if the participant released the key before the change in color of the clock or did not release the key in the go trials $(5.63 \%)$. There were no trials with very short $(<100 \mathrm{~ms})$ response time. Four trials with very long $(>1000 \mathrm{~ms}$ ) response time were excluded from the analysis. In Experiment 3B, the manual response was recorded with a special optical device to avoid any auditory or tactile sensation. Participants held a small piece of stiff felt cloth in their hand that interrupted a horizontal beam of infrared light between an emitter and sensor. When they raised the piece of felt, the sensor signaled that the movement had been made. With this special device, participants could make a hand movement without movement-related auditory or tactile information (and minimal proprioceptive information from the joints and muscles), all of which were more prominent in the key-release task. Trials were also excluded from the analysis if the participant released the sensors in the control condition or no-go trials (8.45 and 30.89\%, respectively). Twenty-two trials in which the participant made more than one hand movement in the go trials were excluded from the analysis. The following were excluded from analysis: $0.10 \%$ with very short $(<100 \mathrm{~ms})$ response time and $0.67 \%$ with very long $(>1000 \mathrm{~ms})$ response time. On the basis of failure to maintain fixation and other errors, $\sim 38 \%$ of the trials in Experiment $3 \mathrm{~A}$ were excluded from the final analyses. Overall, $<10 \%$ of the trials in Experiment 3B were excluded.

\section{Experiment 1}

Participants. Seven naive participants (three males; mean age, 18.7 years; range, $18-19$ years) participated in the study.

Stimuli and procedure. There were two testing sessions consisting of a control condition and a saccade condition presented in an A-B-B-A counterbalanced order. Each condition in the A-B-B-A design consisted of 30 trials, for a total of 240 trials ( 120 control and 120 saccade trials). Before the testing sessions, 30 control and 30 saccade trials were run in a random order for practice. A black fixation dot $\left(1^{\circ}\right.$ in diameter) was presented at $8^{\circ}$ left of center of an otherwise uniformly white screen. Participants initiated a trial by pressing a computer key. A line then appeared with one end attached to the center of the fixation dot and began rotating like a second-hand of a clock (Fig. 1A). The diameter of the clock was $1.8^{\circ}$. One complete rotation took $2560 \mathrm{~ms}$ (Libet et al., 1983). The participants were asked not to track the clock arm. Fixation was monitored online. Figure $1 B$ depicts the time course of the stimuli of Experiment 1. After a variable interval $(2560-3840 \mathrm{~ms})$, the clock turned green [International Commission on Illumination (CIE), $x=0.28, y=$ 0.60 ; mean luminance, $\left.16 \mathrm{~cd} / \mathrm{m}^{2}\right]$, and a black rectangle appeared $8^{\circ}$ right of center of the screen for $40-80 \mathrm{~ms}$. The rectangle was oriented vertically or horizontally. The width and length of the rectangle was $1^{\circ}$ and $2^{\circ}$, respectively. The orientation of the rectangle varied randomly from trial to trial. The probability of occurrence of the vertically oriented rectangle was 0.1 . These trials were excluded from the analysis. In the control condition, the participants were asked to keep looking at the fixation dot. In the saccade condition, the participants were asked to move their eyes to the rectangle. The A-B-B-A counterbalancing was used to ensure that the saccade condition followed the control condition as often as it followed the saccade condition (and vice versa). Whether a participant began the experiment with the saccade condition or with the control condition was randomly determined. After a variable interval (800-1200 $\mathrm{ms}$ ) after the disappearance of the rectangle, the clock turned black again. After the presentation of the target, participants were asked to complete two tasks. The first task was to report the position of the clock hand when the color of the fixation point changed. To do this, participants adjusted the clock hand by pressing a computer key. We called this task the "timing task." The second task was to report the orientation of the rectangle and not to miss any of the vertical orientations, again by pressing a computer key. We called this task the "orientation task." The aim of the orientation task was to force the participants to shift their attention onto the target in both conditions. Trials were excluded from the analysis if the participant made a mistake in the orientation task. After completing these tasks in the saccade condition trials, the participants received feedback about their saccadic latency on that trial. Participants were encouraged to move their eyes as quickly as possible.

\section{Experiment 2}

Participants. Eleven naive participants (three males; mean age, 18.5 years; range, $18-20$ years) were tested.

Stimuli and procedure. There were three testing sessions consisting of a control condition and a saccade condition presented in an A-B-B-A counterbalanced order. Each condition in the A-B-B-A design consisted of 30 trials, for a total of 360 trials. The first 60 trials ( 30 control and 30 saccade trials) were considered as practice trials, and the remaining 300 trials (150 control and 150 saccade trials) were analyzed. Figure $1 C$ depicts the time course of the stimuli of Experiment 2. The procedure of Experiment 2 was identical to that of Experiment 1, except that, in Experiment 2, the clock turned red (CIE, $x=0.63, y=0.32$; mean luminance, $16 \mathrm{~cd} / \mathrm{m}^{2}$ ) on $30 \%$ of the trials in both conditions. In the control condition, the participants were asked to keep looking at the fixation dot. In the go/no-go condition, the participants were asked to move their eyes to the target as quickly as possible if the clock turned green and to keep their eyes on the fixation dot if the clock turned red. The participants performed the timing task and the orientation task as in Experiment 1. When they had completed these two tasks in the go trials, the participants 
A

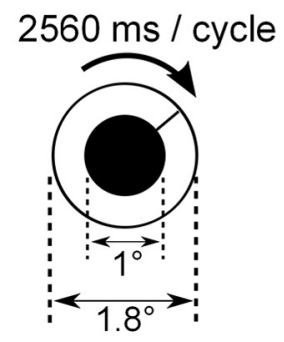

B

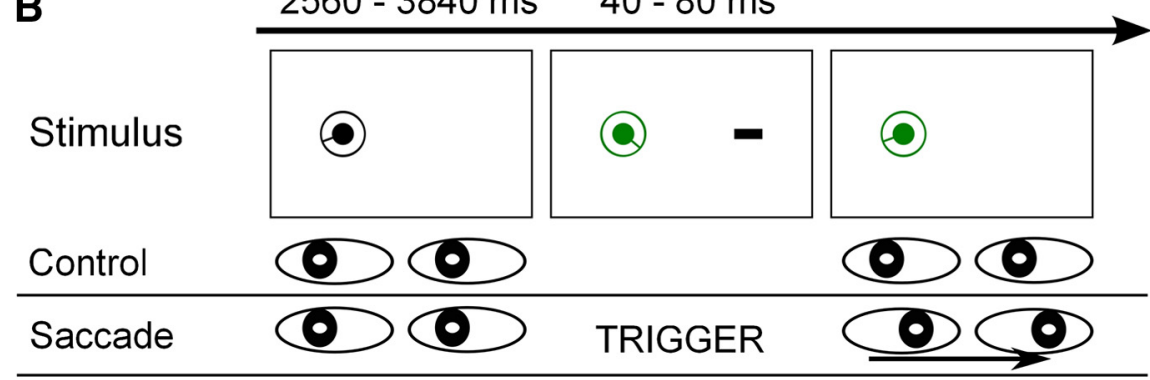

C

Clock

Target

Eye (Control \& No-go)

Eye (Go)

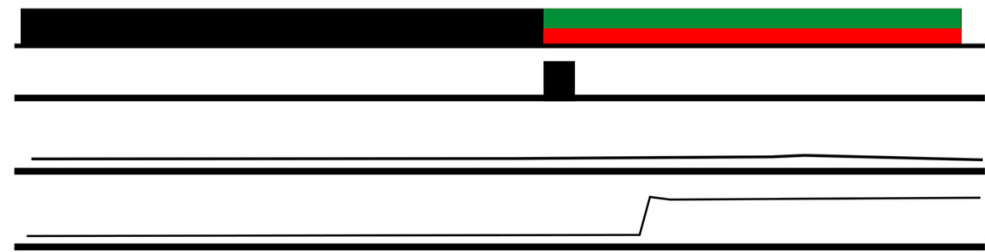

D Clock

Target

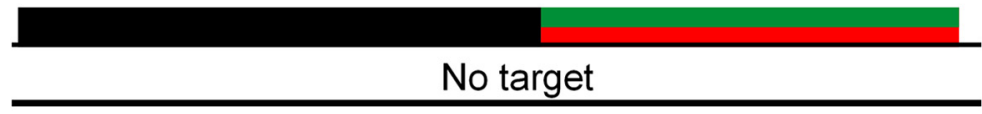

Hand (Control \& No-go)

Hand (Go)

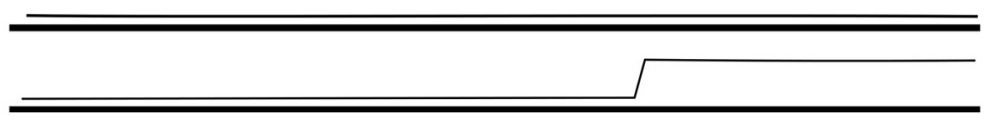

Figure 1. Schematics of experimental methods. $A$, The clock. The fixation dot, the second hand, and the rim circle of our stimulus were presented continuously throughout the trial to avoid the flash-lag effect (Kanai et al., 2009). B, Schematic of Experiment 1. C, Schematic of Experiment 2. D, Schematic of Experiment 3.

were given feedback about whether or not they had made a saccade and what the latency of that saccade was. In the no-go trials, the participants again received feedback about whether they had successfully cancelled the saccade. The participants were asked to make sure they either made or cancelled the saccade correctly and to make the saccade as quickly as possible on go trials.

\section{Experiment $3 A$}

Participants. Thirteen naive participants (four males; mean age, 18.4 years; range, $18-19$ years) were tested. We recruited more participants in this experiment than we did in Experiment 2 because we anticipated that performance on the hand-movement task could be more variable given the presence of sensory feedback from the keyboard that could vary from one individual to another.

Stimuli and procedure. Figure $1 D$ depicts the time course of the stimuli of Experiment 3. The same clock that was used in Experiments 1 and 2 was presented at the center of the screen. The saccade target was not presented. Participants initiated a trial by pressing a computer key and keeping it depressed with their right index finger. The participants were asked to release the key if the clock turned green and not to release the key if the clock turned red. Trials were excluded from the analysis if the participant released the key before the clock changed color. Trials were also excluded from the analysis if the participant released the key in the control condition or depressed the released key again after the first release in the go/no-go condition. The participants wore ear plugs to mask the noise of the key release and used a silent keyboard. Again they reported the perceived position of the second hand of the clock when it changed color. They were asked to use their right index fingers to report the time.

\section{Experiment $3 B$}

Participants. Ten naive participants (one male; mean age, 18.5 years; range, 18-19 years) were tested. Because we reduced the amount of sensory feedback from the hand movement in this experiment by elimi- nating the keyboard, we used the same number of participants as we did in Experiment 2.

Stimuli and procedure. The procedure of Experiment 3B was identical to that of Experiment 3A, except that the participants were required to make a small vertical finger movement that was detected by an optical sensor device instead of a keyboard. Participants held a small piece of stiff felt cloth between the index finger and thumb of their right hand. The piece of felt was positioned so that it interrupted a beam of infrared light between the emitter and sensor of the optical device. Therefore, the sensor could detect when the piece of felt was raised. The same clock that was used in Experiment 3A was presented at the center of the screen. The participants were asked to make a manual response by lifting the piece of felt if the clock turned green and not to make a response if the clock turned red. Trials were excluded from the analysis if the sensors detected a hand movement in the control condition or if the sensors detected more than one hand movement in the go trial. Trials were also excluded from the analysis if the response time was very short $(<100 \mathrm{~ms})$ or very long $(>1000 \mathrm{~ms})$. Again they reported what the perceived position of the second hand of the clock was when it changed color by hitting a computer key with the index finger of the right hand. If necessary, they then picked up the piece of felt in readiness for the next trial.

\section{Statistical analysis}

The deployment of spatial attention between the condition in which participants simply maintained fixation and the condition in which they made a saccade to a target in Experiments 1 and 2 was estimated by three measures: (1) the detection rate of the vertically oriented rectangle target; (2) $d^{\prime}$; and (3) the accuracy in identification of the two target orientations overall. Trials in which the participants misjudged the orientation were excluded from the analysis $(1.57 \%$ of the control condition trials and $1.31 \%$ of the saccade condition trials in Experiment 1, $6.40 \%$ of the control condition trials and $5.53 \%$ of the go/no-go condition trials in 


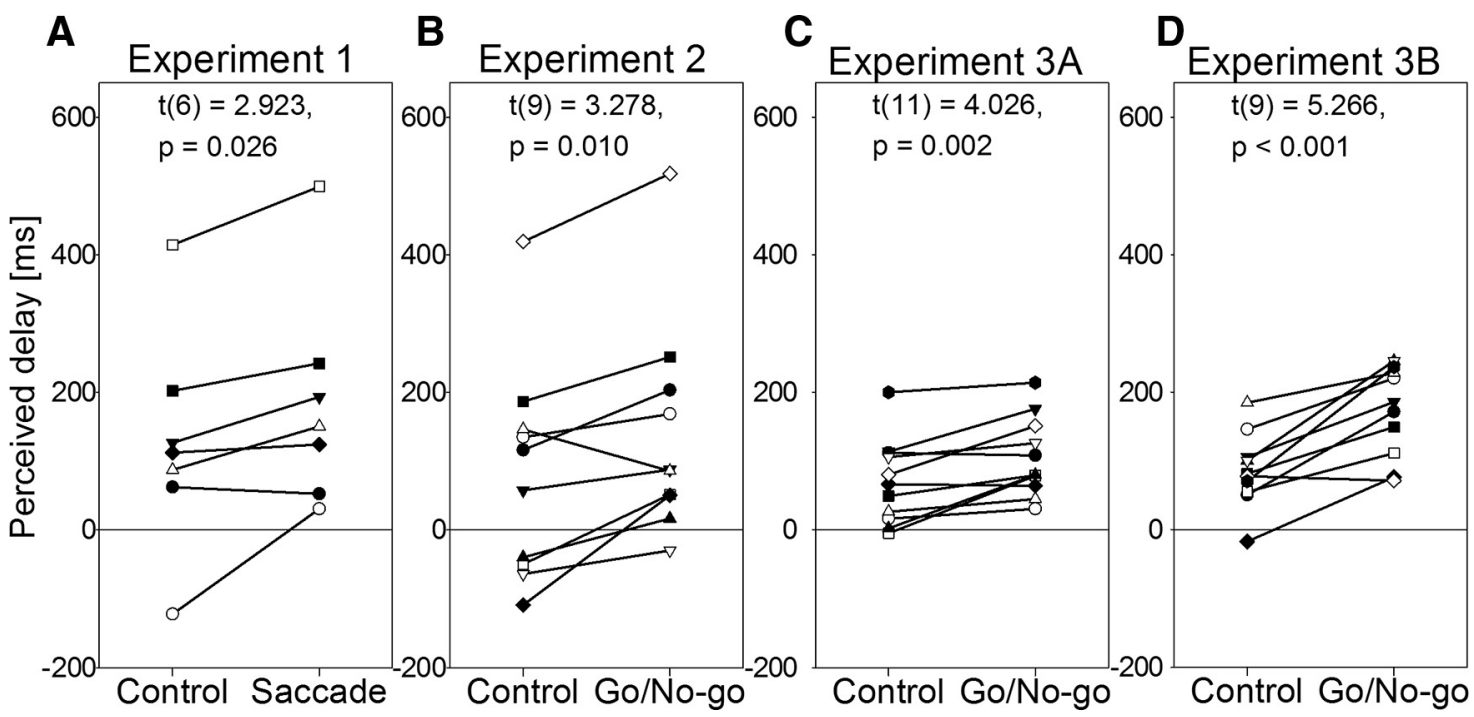

Figure 2. The perceived timing of the change in the color relative to real timing. Each line represents a different participant. $\boldsymbol{A}$, Experiment $1(n=7), \boldsymbol{B}$, Experiment $2(n=10)$. $\boldsymbol{C}$, Experiment $3 \mathrm{~A}(n=12)$. D, Experiment $3 \mathrm{~B}(n=10)$.

Table 1. Results of Experiment 1

\begin{tabular}{|c|c|c|c|c|c|c|c|c|c|c|c|}
\hline \multirow[b]{3}{*}{ Participant } & \multirow{2}{*}{\multicolumn{2}{|c|}{$d^{\prime}$}} & \multicolumn{5}{|c|}{ Perceived delay from the change in color (ms) } & \multirow{2}{*}{\multicolumn{2}{|c|}{ Saccade latency (ms) }} & \multirow{2}{*}{\multicolumn{2}{|c|}{ Correlation }} \\
\hline & & & \multicolumn{2}{|l|}{ Control } & \multicolumn{2}{|l|}{ Saccade } & \multirow{2}{*}{$\frac{\text { Difference from control }}{\text { Saccade }}$} & & & & \\
\hline & Control & Saccade & M & SE & M & SE & & M & SE & $r$ & $p$ \\
\hline 01 & 4.33 & 4.25 & 62.10 & 107.90 & 52.36 & 163.39 & -9.75 & 292.55 & 119.86 & 0.00 & 0.96 \\
\hline 02 & 4.05 & 4.30 & -122.34 & 343.95 & 30.66 & 214.20 & 153.00 & 234.39 & 60.37 & 0.01 & 0.92 \\
\hline 03 & 3.15 & 2.87 & 126.63 & 147.40 & 193.41 & 191.29 & 66.79 & 284.03 & 68.01 & 0.00 & 0.97 \\
\hline 04 & 3.36 & 4.01 & 87.13 & 146.04 & 150.42 & 217.74 & 63.29 & 286.94 & 51.96 & -0.02 & 0.83 \\
\hline 05 & 4.25 & 4.20 & 202.21 & 191.34 & 242.11 & 286.08 & 39.90 & 265.34 & 71.80 & 0.00 & 1.00 \\
\hline 06 & 3.78 & 4.30 & 414.45 & 213.31 & 499.27 & 286.73 & 84.82 & 325.26 & 94.87 & -0.08 & 0.37 \\
\hline 07 & 4.40 & 4.33 & 112.23 & 166.40 & 124.30 & 197.44 & 12.07 & 197.71 & 49.68 & -0.06 & 0.50 \\
\hline M & 3.90 & 4.04 & 126.06 & & 184.65 & & 58.59 & 269.46 & & -0.02 & \\
\hline SE & 0.49 & 0.53 & 56.51 & & 55.03 & & 18.56 & 14.68 & & 0.01 & \\
\hline
\end{tabular}

Experiment 2). The trials with the presentation of the vertically oriented target were excluded from the analysis.

The perceived delay on each trial was calculated by subtracting the real time of the color change from the reported time. One participant from Experiment 2 whose SE of the perceived delay was more than three times larger than other participants in all conditions was excluded from the analysis. One participant from Experiment $3 \mathrm{~A}$ who did not respond in the timing task on most trials was also excluded from the analysis. The mean perceived delay for each condition was calculated for each participant. In Experiment 1, a two-way paired $t$ test was conducted to evaluate the difference in the perceived delay between two conditions. In Experiments 2, 3A, and 3B, ANOVAs were conducted for the perceived delay with the trial types (control, go, and no-go) as the within-subject factor. Post hoc comparisons were based on the Shaffer's modified sequentially rejective Bonferroni's procedure. In addition, two-way paired $t$ tests were conducted to evaluate the difference in the perceived delay between the control and the go/no-go conditions. The Pearson's product-moment correlation between the perceived delay and the latency of the response was calculated for each of the participants.

\section{Results}

\section{Experiment 1}

To control for deployment of spatial attention between the condition in which participants simply maintained fixation and the condition in which they made a saccade to a target, we asked participants to report the orientation of the target. The probability of occurrence of the vertically oriented rectangle was 0.1 and that of the horizontally oriented one was 0.9 . The mean detection rate for the vertically oriented rectangle was 0.97 (range, $0.89-1$ ) in the control condition and 0.92 (range, $0.64-1$ ) in the saccade condition. There was no difference in the detection rate for the vertically oriented target between the two conditions. There was also no difference in $d^{\prime}$ or accuracy in identification of the two target orientations overall, suggesting that, in both conditions, the participants were attending to the target. Nevertheless, the perceived timing of the color change in the clock was significantly later in the saccade condition than it was in the control condition $\left(t_{(6)}=2.922, p=0.027\right.$; Fig. $2 A$; Table 1$)$, suggesting a delay in the perceived timing of sensory events that trigger saccades. [This difference was still significant when the participant with a detection rate of 0.64 for the vertically oriented target was excluded $(p=0.033)$.] The mean delay for the seven participants was 59 ms. What caused this delay? Was it the act of moving the eyes or was it the programming of the movement?

\section{Experiment 2}

To test whether the saccade had to be executed to distort time perception or whether programming of the saccade without actual execution was sufficient, we performed a second experiment in which the clock turned green on $70 \%$ of the trials and red on the remaining $30 \%$. In addition to this go/no-go task, we also included the same control task that we used in our first experi- 
Table 2. Results of Experiment 2

\begin{tabular}{|c|c|c|c|c|c|c|c|c|c|c|c|c|c|c|c|c|c|}
\hline \multirow[b]{3}{*}{ Participant } & \multirow{2}{*}{\multicolumn{2}{|c|}{$d^{\prime}$}} & \multicolumn{11}{|c|}{ Perceived delay from the change in color (ms) } & \multirow{2}{*}{\multicolumn{2}{|c|}{ Saccade latency (ms) }} & \multirow{2}{*}{\multicolumn{2}{|c|}{ Correlation }} \\
\hline & & & \multicolumn{2}{|l|}{ Control } & \multicolumn{2}{|l|}{ Go/no-go } & \multicolumn{2}{|l|}{ Go } & \multicolumn{2}{|l|}{ No-go } & \multicolumn{3}{|c|}{ Difference from control } & & & & \\
\hline & Control & Go/no-go & M & SE & M & SE & M & SE & M & SE & Go/no-go & Go & No-go & M & SE & $r$ & $p$ \\
\hline 08 & 4.27 & 3.63 & 116.02 & 88.80 & 203.18 & 118.65 & 205.02 & 108.66 & 195.43 & 157.33 & 87.16 & 89.00 & 79.42 & 287.80 & 61.57 & -0.17 & 0.09 \\
\hline 09 & 4.01 & 3.92 & 134.61 & 97.11 & 168.41 & 111.81 & 168.86 & 109.33 & 167.13 & 120.25 & 33.79 & 34.25 & 32.52 & 296.52 & 69.41 & -0.03 & 0.78 \\
\hline 10 & 3.67 & 3.88 & 57.54 & 121.15 & 87.44 & 190.83 & 63.23 & 188.75 & 173.08 & 175.94 & 29.90 & 5.69 & 115.54 & 268.34 & 72.03 & 0.08 & 0.40 \\
\hline 11 & 1.81 & 2.30 & 146.18 & 244.98 & 85.50 & 351.35 & 55.57 & 356.56 & 158.34 & 332.84 & -60.68 & -90.61 & 12.16 & 324.11 & 97.54 & 0.15 & 0.13 \\
\hline 12 & 2.64 & 2.99 & 186.46 & 137.01 & 251.22 & 201.48 & 248.13 & 206.65 & 277.18 & 157.44 & 64.76 & 61.67 & 90.72 & 270.43 & 73.61 & -0.13 & 0.17 \\
\hline 06 & 3.64 & 2.87 & -49.85 & 116.82 & 51.32 & 271.50 & 38.38 & 290.81 & 97.48 & 185.02 & 101.17 & 88.22 & 147.33 & 331.60 & 82.25 & -0.04 & 0.67 \\
\hline 13 & 3.78 & 3.67 & -109.22 & 144.06 & 50.22 & 174.59 & 91.17 & 176.79 & -71.29 & 93.64 & 159.43 & 200.39 & 37.93 & 313.89 & 72.36 & -0.26 & 0.01 \\
\hline 14 & 3.59 & 3.83 & 419.21 & 234.33 & 518.30 & 206.55 & 527.89 & 204.34 & 487.78 & 214.47 & 99.09 & 108.68 & 68.57 & 353.85 & 88.63 & -0.06 & 0.57 \\
\hline 15 & 3.61 & 3.48 & -39.77 & 168.72 & 16.66 & 128.23 & 35.19 & 130.87 & -40.79 & 101.62 & 56.43 & 74.97 & -1.02 & 324.66 & 72.38 & 0.06 & 0.55 \\
\hline 16 & 2.30 & 2.82 & -64.06 & 153.13 & -29.75 & 332.95 & -64.38 & 334.83 & 137.66 & 274.26 & 34.31 & -0.32 & 201.72 & 324.87 & 103.11 & 0.06 & 0.57 \\
\hline M & 3.33 & 3.34 & 79.71 & & 140.25 & & 136.91 & & 158.20 & & 60.54 & 57.19 & 78.49 & 309.61 & & -0.03 & \\
\hline SE & 0.80 & 0.55 & 47.26 & & 47.54 & & 49.56 & & 46.99 & & 17.52 & 23.19 & 18.98 & 8.38 & & 0.04 & \\
\hline
\end{tabular}

Table 3. Results of Experiment 3A

\begin{tabular}{|c|c|c|c|c|c|c|c|c|c|c|c|c|c|c|c|}
\hline \multirow[b]{3}{*}{ Participant } & \multicolumn{11}{|c|}{ Perceived delay from the change in color (ms) } & & & & \\
\hline & \multicolumn{2}{|l|}{ Control } & \multicolumn{2}{|c|}{ Go/no-go } & \multicolumn{2}{|l|}{ Go } & \multicolumn{2}{|l|}{ No-go } & \multicolumn{3}{|c|}{ Difference from control } & \multicolumn{2}{|c|}{ Response latency (ms) } & \multicolumn{2}{|c|}{ Correlation } \\
\hline & M & SE & M & SE & $M$ & SE & $M$ & SE & Go/no-go & Go & No-go & M & SE & $r$ & $p$ \\
\hline 17 & 112.20 & 136.34 & 108.27 & 101.57 & 120.38 & 96.13 & 77.00 & 109.98 & -3.94 & 8.18 & -35.20 & 361.51 & 75.22 & 0.19 & 0.05 \\
\hline 18 & 16.25 & 281.53 & 30.46 & 300.71 & -10.51 & 203.80 & 163.63 & 485.24 & 14.22 & -26.76 & 147.38 & 542.51 & 141.89 & -0.17 & 0.09 \\
\hline 19 & 113.58 & 154.30 & 176.10 & 146.77 & 139.28 & 122.99 & 187.78 & 153.05 & 62.52 & 25.70 & 74.19 & 476.82 & 174.03 & -0.02 & 0.87 \\
\hline 20 & 26.22 & 121.02 & 44.82 & 120.22 & 48.24 & 85.07 & 35.22 & 189.64 & 18.60 & 22.02 & 9.00 & 360.08 & 100.19 & -0.03 & 0.77 \\
\hline 21 & 49.22 & 100.13 & 79.45 & 134.96 & 73.59 & 135.99 & 98.21 & 132.56 & 30.23 & 24.37 & 48.99 & 482.78 & 119.89 & -0.02 & 0.81 \\
\hline 22 & -4.96 & 214.23 & 78.51 & 227.92 & 58.78 & 141.68 & 105.83 & 311.95 & 83.47 & 63.74 & 110.79 & 331.84 & 62.51 & 0.08 & 0.42 \\
\hline 23 & 66.35 & 144.82 & 63.97 & 221.05 & 73.56 & 139.50 & 46.33 & 324.86 & -2.37 & 7.22 & -20.01 & 434.24 & 109.14 & -0.06 & 0.53 \\
\hline 24 & 80.14 & 115.23 & 151.16 & 164.10 & 131.18 & 109.18 & 231.08 & 286.66 & 71.02 & 51.04 & 150.94 & 341.10 & 74.88 & 0.09 & 0.36 \\
\hline 25 & 2.15 & 121.63 & 79.88 & 134.01 & 37.13 & 113.93 & 149.63 & 137.73 & 77.73 & 34.98 & 147.48 & 407.96 & 101.88 & 0.03 & 0.78 \\
\hline 26 & 105.80 & 178.71 & 126.23 & 133.01 & 124.53 & 139.57 & 131.35 & 113.17 & 20.43 & 18.72 & 25.55 & 377.51 & 102.63 & 0.06 & 0.56 \\
\hline 27 & 199.95 & 221.67 & 214.13 & 204.69 & 242.08 & 136.02 & 102.32 & 359.88 & 14.18 & 42.13 & -97.63 & 404.67 & 131.83 & 0.05 & 0.62 \\
\hline 28 & 107.35 & 129.38 & 155.74 & 202.25 & 130.59 & 198.82 & 240.62 & 194.22 & 48.39 & 23.24 & 133.27 & 317.92 & 70.09 & -0.07 & 0.46 \\
\hline M & 72.85 & & 109.06 & & 97.40 & & 130.75 & & 36.21 & 24.55 & 57.90 & 403.24 & & 0.01 & \\
\hline SE & 16.40 & & 15.54 & & 17.97 & & 18.34 & & 8.61 & 6.40 & 22.95 & 19.12 & & 0.03 & \\
\hline
\end{tabular}

ment. Notice that participants would have to suppress the tendency to make a saccade much more on the no-go trials than on the control trials. In other words, a saccade to the target would be programmed on both go and no-go trials, although the actual execution of the saccade would occur only on go trials.

Again, we calculated the detection rate for the vertically oriented rectangle. The mean rate was 0.97 (range, 0.82-1) in the control condition and 0.92 (range, $0.79-1$ ) in the saccade condition. There was no difference in the $d^{\prime}$ or the detection rate, and again there was no difference in accuracy in identification of the two target orientations.

The one-way ANOVA revealed a significant effect of conditions on perceived timing $\left(F_{(2,18)}=4.759, p=0.022\right)$. The post hoc tests revealed that the perceived timing of the change in color relative to the real timing was larger in both the go and the no-go trials than it was in the control condition $\left(t_{(9)}=2.339, p=0.044\right.$ and $t_{(9)}=3.923, p=0.011$, respectively). The mean delay, compared with the control condition, was $57 \mathrm{~ms}$ for the go trials and $78 \mathrm{~ms}$ for the no-go trials. There was no difference in the perceived timing of the color change between go and no-go trials $\left(t_{(9)}=0.648, p=0.533\right)$. These results are summarized in Figure $2 B$ and Table 2 . In short, the fact that the same delay in the perception of the triggering event occurred on both go and no-go trials suggests that the temporal distortion is related to action programming, not the action itself. Moreover, this also shows that the blurring of the image during the saccade cannot explain the temporal distortion.

\section{Experiment 3A}

Next, we examined whether or not the perceived temporal shift of the triggering event is saccade specific. In a third experiment, the clock was presented at the center of the screen and a target was never presented. Instead of making a saccade, the participants were instructed to release a key that they were holding down with their right index finger if the clock turned green but were instructed not to release the key if the clock turned red (Fig. 1D). The participants wore ear plugs to mask the noise of the key release. Again they reported the perceived position of the second hand of the clock when it changed color.

The one-way ANOVA revealed a significant effect of conditions $\left(F_{(2,22)}=4.15, p=0.03\right)$. The post hoc tests revealed that the perceived timing of the change in the color relative to the real timing was larger in both the go and the no-go trials than it was in the control condition $\left(t_{(11)}=2.415, p=0.034\right.$ and $t_{(11)}=3.672$, $p=0.011$, respectively). The mean delay, compared with the control condition, was $25 \mathrm{~ms}$ for the go trials and $58 \mathrm{~ms}$ for the no-go trials. There was a small difference in the perceived timing of the color change between the go and no-go trials, but it failed to reach significance $\left(t_{(11)}=1.361, p=0.201\right)$. These results are summarized in Figure $2 \mathrm{C}$ and Table 3. 
Table 4. Results of Experiment 3B

\begin{tabular}{|c|c|c|c|c|c|c|c|c|c|c|c|c|c|c|c|}
\hline \multirow[b]{3}{*}{ Participant } & \multicolumn{11}{|c|}{ Perceived delay from the change in color (ms) } & & & & \\
\hline & \multicolumn{2}{|l|}{ Control } & \multicolumn{2}{|c|}{ Go/no-go } & \multicolumn{2}{|l|}{ Go } & \multicolumn{2}{|l|}{ No-go } & \multicolumn{3}{|c|}{ Difference from control } & \multicolumn{2}{|c|}{ Response latency (ms) } & \multicolumn{2}{|c|}{ Correlation } \\
\hline & M & SE & M & SE & M & SE & M & SE & Go/no-go & Go & No-go & M & SE & $r$ & $p$ \\
\hline 30 & 146.15 & 16.87 & 220.35 & 17.09 & 239.20 & 16.04 & 145.69 & 54.64 & 74.20 & 93.04 & -0.46 & 439.60 & 11.93 & 0.34 & 0.00 \\
\hline 31 & 105.52 & 10.43 & 185.64 & 10.86 & 188.99 & 11.00 & 174.82 & 29.44 & 80.11 & 83.47 & 69.29 & 426.87 & 9.18 & 0.06 & 0.46 \\
\hline 32 & 184.75 & 24.34 & 227.70 & 28.13 & 189.88 & 30.02 & 337.92 & 64.08 & 42.95 & 5.13 & 153.18 & 565.02 & 17.02 & -0.22 & 0.01 \\
\hline 35 & -17.14 & 10.36 & 76.36 & 12.29 & 78.81 & 15.53 & 68.77 & 15.11 & 93.49 & 95.95 & 85.91 & 435.84 & 11.42 & 0.05 & 0.52 \\
\hline 36 & 77.70 & 9.60 & 71.31 & 12.30 & 74.96 & 13.63 & 61.60 & 26.98 & -6.38 & -2.74 & -16.09 & 469.58 & 7.61 & -0.02 & 0.78 \\
\hline 37 & 100.36 & 10.53 & 245.36 & 21.43 & 263.92 & 16.72 & 147.96 & 100.54 & 145.01 & 163.56 & 47.60 & 361.64 & 8.81 & 0.17 & 0.03 \\
\hline 38 & 70.61 & 8.48 & 236.57 & 18.15 & 263.08 & 18.61 & 157.03 & 44.16 & 165.96 & 192.47 & 86.42 & 547.27 & 14.05 & 0.12 & 0.14 \\
\hline M & 85.51 & & 169.51 & & 175.32 & & 142.79 & & 84.00 & 89.80 & 57.27 & 458.82 & & 0.07 & \\
\hline SE & 16.47 & & 19.65 & & 21.04 & & 23.66 & & 15.13 & 18.91 & 14.30 & 19.63 & & 0.04 & \\
\hline
\end{tabular}

\section{Experiment 3B}

In Experiment 3A, we found that participants again perceived a delay in the timing of the triggering event in a manual response task. However, this temporal delay was somewhat smaller than it was in the saccade task, at least for the go trials. Indeed, in the manual response task, the temporal delay on go trials was somewhat smaller (although not significantly so) than it was on no-go trials. Why should this be the case for the manual but not the eye-movement response? In the key-release task, it is possible that there were a number of movement-related sensory changes in the environment, both tactile and auditory in nature (although the perceived noise of the keyboard was reduced by ear plugs). In the no-go trials of the manual response task, these movementlinked sensory changes would be absent. Experiment 3B was designed as a replication of Experiment $3 \mathrm{~A}$ but with a manual response that resulted in far less movement-related sensory information. The procedure was identical to that of Experiment $3 \mathrm{~A}$ except that, instead of a keyboard, an optical sensor was used to measure the response time of hand movement.

The one-way ANOVA revealed a significant effect of conditions $\left(F_{(2,18)}=9.862, p=0.001\right)$. The post hoc tests revealed that the perceived timing of the change in the color relative to the real timing was larger in both the go and no-go trials than it was in the control condition $\left(t_{(9)}=4.505, p=0.004\right.$ and $t_{(9)}=3.800, p=0.008$, respectively). The mean delay, compared with the control condition, was $90 \mathrm{~ms}$ for the go trials and $57 \mathrm{~ms}$ for the no-go trials. Again, there was no difference in the perceived timing of the color change between the go and no-go trials $\left(t_{(9)}=1.293, p=0.228\right)$. These results are summarized in Figure $2 D$ and Table 4 . In short, a temporal delay of the same magnitude occurred in both go and no-go trials when the movement-related sensory information was reduced.

\section{Discussion}

In these experiments, we showed that the perceived timing of a sensory event that triggered an action was significantly delayed compared with the perceived timing of an identical event that was simply observed passively. Moreover, we found that this was the case even if the actions were eventually cancelled on no-go trials, suggesting that the temporal distortion was not related to the action itself but to the programming of that action.

\section{The effect of attention}

One might argue that the temporal shift we observed was attributable to differences in the deployment of spatial attention and not to differences in the action requirements of the experimental and the control tasks. Simply put, it might have been the case that the participants had to attend to the location of the target when making a saccade, and thus the processing of change in color of the clock may have been delayed. However, in both Experiments 1 and 2, participants had to report the time at which the color of the fixation point changed and the orientation of the target in both the experimental and the control tasks. In other words, the experimental and the control tasks had similar attentional requirements. More importantly, the temporal shift was also observed in the hand-movement task in which there was no peripheral target and participants kept their eyes and attention on the fixation point. Thus, it is highly unlikely that the temporal shift we observed reflected differences in the deployment of spatial attention.

Alternatively, one might argue that participants attended to the clock more in the experimental conditions than in control conditions because the participants needed to react to the change in color as soon as possible in the experimental conditions. This, too, seems unlikely. Previous studies have shown that spatial attention increases the speed of information transmission (Stelmach and Herdman, 1991; Hikosaka et al., 1993a,b, 1996; Shore et al., 2001). Thus, it is not reasonable to suggest that the more we attend to an event, the later it would seem to occur. In fact, if anything, attending to the clock more on experimental than control trials might actually reduce the apparent delay in the perceived onset of triggering event.

One might still argue that the apparent temporal delay on action trials arose because participants attended to the clock only after they had initiated the action. However, if this were true, then the size of delay should be correlated with the latency of the action. As Tables 1-4 show, this is not the case.

A skeptic could still argue that a difference in cognitive load between the experimental and control trials could have led to the difference in perceived timing of the stimuli: that is, the pressure to respond quickly to the imperative stimulus could have delayed attending to the clock and thus the perceived timing of the stimulus. However, if that were true, one would expect that the perceived timing of the stimulus in Experiment 1 would be earlier than the perceived timing of the stimulus on the go trials of Experiment 2, in which the cognitive load was even higher because participants had to decide whether or not to respond to the stimulus. As it turned out, this was not the case: the mean perceived delay was $184.65 \mathrm{~ms}$ for the experimental trials in Experiment 1 and $136.91 \mathrm{~ms}$ for the go trials in Experiment 2, a nonsignificant difference in the opposite direction from what a cognitive load explanation would predict. 


\section{Flash-lag effect}

Although it might be argued that apparent delay in the perceived position of the moving second hand was attributable to some sort of action-related enhancement of a flash-lag effect, we believe that this is unlikely. The flash-lag effect depends on the sudden onset of a new stimulus (Kanai et al., 2009). In the present study, the fixation dot, the moving second hand, and surrounding circle were continuously present throughout the trial, thus obviating the occurrence of a flash-lag effect. The saccadic target itself was $16^{\circ}$ away from the clock and was therefore unlikely to have induced a flash-lag effect. Moreover, we also observed the same temporal delay in Experiments $3 \mathrm{~A}$ and $3 \mathrm{~B}$, in which there was no peripheral target at all. In short, we would argue that the flash-lag effect was not operating in our experiments.

\section{Effect of action execution}

It is also unlikely that the temporal shift was attributable to the execution of the action per se. The size of the temporal distortion was not correlated with saccadic or hand-movement latency. In short, the awareness of the timing of the triggering event was not linked to the timing of the motor response. Moreover, this conclusion is bolstered by the fact that the same temporal shift was observed in the no-go trials in which no motor response was made.

\section{Intentional binding by movement-related sensory information} The temporal shift observed in Experiment 3A was somewhat smaller than the shift observed in the saccade task. In the keyrelease task, unlike the eye-movement task, there would be movement-related proprioceptive information from the skin, joints, and muscles, possible auditory stimulation (although the noise of the keyboard was masked by ear plugs), and changes in tactile input. Sensory events triggered by a voluntary action have been shown to be perceived earlier in time than nontriggered events, suggesting that voluntary actions are bound temporally to their perceived effects (Haggard et al., 2002; Haggard and Clark, 2003; Wohlschläger et al., 2003; Buehner and Humphreys, 2009; Kawabe et al., 2013). Thus, the proprioceptive and other sensory feedback that signals the effect of a manual action might promote the binding between the intention to act and the effect of the action and reduce the binding between the event that triggers the action and the triggered intention to act. This conclusion is strengthened by the fact that the temporal delay observed in the no-go trials in the key-release task, in which proprioceptive feedback and other sensory changes would be absent, was somewhat larger than it was in the go trials. This conclusion is also supported by the results of Experiment $3 \mathrm{~B}$ in which the movement-related sensory information was reduced substantially. In this experiment, the temporal delay on go trials was virtually identical to what it was on the no-go trials and was now quite similar in magnitude to the temporal delay observed in the eye-movement experiments.

\section{Sense of agency with action preparation}

As discussed earlier, previous studies have focused on the perceived onset of stimulus events that are triggered by self-paced actions (Haggard et al., 2002; Haggard and Clark, 2003; Wohlschläger et al., 2003; Buehner and Humphreys, 2009; Kawabe et al., 2013). Because the stimulus events that follow these actions are perceived to occur earlier in time than events that are not preceded by such actions, it has been argued that some sort of temporal binding occurs between actions and the effects of those actions. The binding is thought to depend on the subjective experience one has of causing the effect by one's own actions (i.e., a sense of agency). Events that are perceived not to be caused by one's actions are not subject to the temporal distortion because there is no binding between one's actions and the unrelated events. Our results challenge this conclusion. In our case, the stimulus events triggered the actions rather than the other way round. Nevertheless, the perceived onset of the stimulus event was moved closer in time to the action it triggered. Therefore, one might conclude that a temporal binding has occurred between the event and the triggered action. However, notice that one cannot invoke a sense of agency to explain such binding. After all, the event was not triggered by an action. Quite the opposite: the action was triggered by the event. Moreover, the temporal delay in the perceived timing of the triggering event occurred even when the actions were cancelled. In other words, temporal binding occurred between a triggering event and the programming on an action (without actual execution), a situation in which a sense of agency in the way it is usually conceived cannot operate at all. A cancelled action has (by definition) no effect on the world. Of course, it is possible that having a sense of agency over one's actions could work backward in time and could bind those actions to the events that triggered them. After all, these actions were not reflexive; people could decide (and they did on no-go trials) not to react to the stimulus. Perhaps this is sufficient to bind the triggering event to the action. In any case, any explanation for temporal binding that invokes the sense of agency needs to be carefully reexamined. It may be the case that temporal binding operates both forward and backward in time with respect to voluntary actions, helping us to identify the causal links between the world of sensory events and our actions in that world.

\section{References}

Brainard DH (1997) The Psychophysics Toolbox. Spat Vis 10:433-436. CrossRef Medline

Buehner MJ, Humphreys GR (2009) Causal binding of actions to their effects. Psychol Sci 20:1221-1228. CrossRef Medline

Haggard P, Chambon V (2012) Sense of agency. Curr Biol 22:R390-R392. CrossRef Medline

Haggard P, Clark S (2003) Intentional action: conscious experience and neural prediction. Conscious Cogn 12:695-707. CrossRef Medline

Haggard P, Clark S, Kalogeras J (2002) Voluntary action and conscious awareness. Nat Neurosci 5:382-385. CrossRef Medline

Hikosaka O, Miyauchi S, Shimojo S (1993a) Voluntary and stimulusinduced attention detected as motion sensation. Perception 22:517-526. CrossRef Medline

Hikosaka O, Miyauchi S, Shimojo S (1993b) Focal visual attention produces illusory temporal order and motion sensation. Vision Res 33:1219-1240. CrossRef Medline

Hikosaka O, Miyauchi S, Shimojo S (1996) Orienting a spatial attention-its reflexive, compensatory, and voluntary mechanisms. Brain Res Cogn Brain Res 5:1-9. CrossRef Medline

Kanai R, Carlson TA, Verstraten FAJ, Walsh V (2009) Perceived timing of new objects and feature changes. J Vis 9:5. CrossRef Medline

Kawabe T, Roseboom W, Nishida S (2013) The sense of agency is action effect causality perception based on cross-modal grouping. Proc R Soc B 280:20130991. CrossRef Medline

Libet B, Gleason CA, Wright EW, Pearl DK (1983) Time of conscious intention to act in relation to onset of cerebral activity (readiness-potential). The unconscious initiation of a freely voluntary act. Brain 106:623-642. CrossRef Medline

Pelli DG (1997) The VideoToolbox software for visual psychophysics: transforming umbers into movies. Spat Vis 10:437-442. CrossRef Medline

Shore DI, Spence C, Klein RM (2001) Visual prior entry. Psychol Sci 12: 205-212. CrossRef Medline

Stelmach LB, Herdman CM (1991) Directed attention and perception of temporal order. J Exp Psychol Hum Percept Perform 17:539-550. CrossRef Medline

Wohlschläger A, Haggard P, Gesierich B, Prinz W (2003) The perceived onset time of self- and other-generated actions. Psychol Sci 14:586-591. CrossRef Medline 